\title{
Time variation of SWS spectra of M-type Mira variables
}

\section{Z Cyg and its dust optical properties}

\author{
T. Onaka ${ }^{1}$, T. de Jong ${ }^{2,3}$, and I. Yamamura ${ }^{4}$ \\ 1 Department of Astronomy, Graduate School of Science, University of Tokyo, Tokyo 113-0033, Japan \\ 2 SRON, Sorbonnelaan 2, 3584 CA Utrecht, The Netherlands \\ 3 Astronomical Institute Anton Pannekoek, University of Amsterdam, 1098 SJ Amsterdam, The Netherlands \\ 4 Institute of Space and Astronautical Science, Sagamihara, Kanagawa 229-8510, Japan
}

Received 30 January 2002 / Accepted 11 April 2002

\begin{abstract}
The M-type Mira variable star, Z Cyg, was observed with the Short-Wavelength Spectrometer (SWS) on board the Infrared Space Observatory (ISO) 7 times at roughly 60 day intervals over one and a half period. The infrared spectrum $(2.38-45.2 \mu \mathrm{m})$ of Z Cyg shows prominent silicate emission bands at $10 \mu \mathrm{m}$ and $18 \mu \mathrm{m}$ and displays quite large variations over the observed period. The variation in the infrared spectrum of $\mathrm{Z}$ Cyg is synchronized with the visual light curve. The circumstellar emission and the $10 \mu \mathrm{m}$ to $18 \mu \mathrm{m}$ silicate band ratio increases at maximum and decreases at minimum, indicating a variation in the dust temperature with phase. Apart from minor emission features which may be partly due to oxide dust, the observed spectra can be fitted by optically thin dust shell models with one single silicate dust emissivity profile. Thus silicate is the dominant dust component in the circumstellar shell of Z Cyg. The variation in the integrated infrared flux and in the dust temperature derived from the observed spectra can be interpreted in terms of the variation in the luminosity of the central star if proper dust optical properties are adopted. Conversely, the dust emissivity can be estimated from the variations in the infrared spectrum. The derived optical properties are relatively insensitive to the assumptions made in the model analysis because of the optically thin nature of the dust shell. Possible evidence for dust formation near minimum is discussed. The observed variation of the dust shell spectrum of $\mathrm{Z}$ Cyg is fitted most consistently with a model in which the inner dust shell temperature is $700 \pm 100 \mathrm{~K}$ at maximum. From the model fits we derive a ratio of the $18 \mu \mathrm{m}$ and $10 \mu \mathrm{m}$ emission efficiencies of $Q(18 \mu \mathrm{m}) / Q(10 \mu \mathrm{m})=0.51 \pm 0.08$. The $\mathrm{Z}$ Cyg dust also has a broad feature in the $20-25 \mu \mathrm{m}$ region in addition to the $18 \mu \mathrm{m}$ silicate band. The optical properties of dust grains around Z Cyg are compared with those in circumstellar shells of other oxygen-rich late-type stars and it is shown that there are variations in the $20 \mu \mathrm{m}$ emissivity of circumstellar dust, possibly related to the presence of another dust component.
\end{abstract}

Key words. stars: AGB and post-AGB - (stars:) circumstellar matter - stars: individual: Z Cyg stars: mass-loss - (ISM:) dust, extinction - infrared: stars

\section{Introduction}

The IRAS LRS database has revealed a large variety in the mid-infrared spectra, particularly in the $10 \mu \mathrm{m}$ region, of M-type Mira variable stars (Cheeseman et al. 1989; LittleMarenin \& Little 1988, 1990; Simpson 1991). Stencel et al. (1990) discussed the diversity in terms of the time evolution of the dust shell, while Sloan \& Price $(1995,1998)$

Send offprint requests to: T. Onaka,

e-mail: onaka@astron.s.u-tokyo.ac.jp

* Based on observations with ISO, an ESA project with instruments funded by ESA Member States (especially the PI countries: France, Germany, the Netherlands and the United Kingdom) and with the participation of ISAS and NASA. proposed a classification scheme of the mid-infrared spectra with the silicate index following the classification by Little-Marenin \& Little $(1988,1990)$. Onaka et al. (1989a) and Miyata et al. (2000) have shown that the dust shell spectra can be decomposed at least into two components, silicate and aluminum oxide. Speck et al. (2000) have studied the diversity in more detail by recent ground-based observations of a large number of AGB stars. The variety in the observed dust features suggests a complex dust formation process in oxygen-rich Mira variable stars (cf. Ferrarotti \& Gail 2001).

Dust grains are formed in the mass-loss outflow which is accelerated by the radiation pressure on the grains. The pulsation has been suggested to play an important role 
in the mass loss and dust formation process (Vardya et al. 1986). Theoretical investigations indicate complicated interactions between dust formation and mass-loss processes in pulsating stars (Winters et al. 1994, 2000; Höfner \& Dorfi 1997; Höfner et al. 1998; Simis et al. 2001). The light variation affects the incident energy on the circumstellar dust shell, while dust formation may be modulated by the variability. Thus the study of the variability in the infrared spectrum of the dust shell gives insight into the mass loss and dust formation process as well as the optical properties of circumstellar dust grains.

Past studies of the infrared variability in oxygen-rich Mira variables based on the IRAS LRS database suggested an enhancement of the silicate emission around the visual maximum for a few Mira variable stars (Hron \& Aringer 1994; Little-Marenin et al. 1996). Groundbased photometric observations indicated a similar trend (Le Betre 1993). However, the IRAS observations were not well suited for periodic monitoring of variable stars and hampered as they were by limited temporal coverage. Creech-Eakman et al. (1997) have suggested variations in the $10 \mu \mathrm{m}$ silicate feature of several long-period variable (LPV) stars by comparing ground-based observations with the IRAS LRS spectra. After decomposing the $10 \mu \mathrm{m}$ region spectra of 18 oxygen-rich Mira variables into two components, Miyata (1998) has suggested that only the silicate component shows a clear variation with the visual variability based on the comparison between groundbased observations and the IRAS LRS spectra. Possible long-term variations, however, make it difficult to directly compare the data taken at nearly a decade apart (Monnier et al. 1999). With the recent development of mid-infrared instruments for ground-based telescopes Monnier et al. (1998) have clearly shown by ground-based spectroscopy that there are different variability types of LPVs in the $10 \mu \mathrm{m}$ region, and indicated that a class of stars show an enhancement in the $10 \mu \mathrm{m}$ silicate emission feature around the visual maximum. Model calculations by Lobel et al. (2000) have indicated that the variation seen in the $10 \mu \mathrm{m}$ band can be caused by the modulation of the mass-loss rate due to the luminosity change of the central star.

While the accessible spectral range in the mid-infrared from the ground is limited, a wide spectral coverage in the 10 to $20 \mu \mathrm{m}$ region is crucial for the study of dust shell properties as demonstrated by IRAS LRS studies (e.g., Bedijn 1987; Volk \& Kwok 1988; Onaka et al. 1989a; Simpson 1991). We have carried out periodic observations of infrared variability in two M-type Mira variable stars with the Short-Wavelength Spectrometer (SWS; de Graauw et al. 1996) on board the Infrared Space Observatory (ISO; Kessler et al. 1996) to investigate the time variability of their infrared spectra free from the terrestrial atmospheric disturbance (Onaka et al. 1999, TONAKA.TIMVAR and VARSPC). In this paper we present the results of observations and analysis of the infrared variability in one of the target stars, Z Cyg.

In a preliminary study Onaka et al. (1999) have shown that all of the Z Cyg spectra taken at different variability phases were fitted fairly well by simple models of optically thin dust shell with the same dust emissivity with different inner dust shell temperatures and densities. While the variation in the inner dust shell temperatures may be interpreted in terms of the central star luminosity variation, the variation in the density is difficult to account for since it requires changes in the amount of dust in the entire dust shell.

The derived mass-loss rate is a function of the inner dust shell temperature, which is then a function of the adopted dust optical properties. In the previous work by Onaka et al. (1999), the optical properties were derived from the observed spectrum at maximum by assuming rather arbitrarily that the inner dust shell temperature was set as $800 \mathrm{~K}$ and that the dust temperature was given by a power law of the distance from the star. A key parameter to determine the dust temperature from the observed spectra is the ratio of the $18 \mu \mathrm{m}$ to $10 \mu \mathrm{m}$ bands in the dust emissivity. Several optical properties have been proposed and investigated for silicate grains (Draine \& Lee 1984; Bedijn 1987; Volk \& Kwok 1988; Simpson 1991; David \& Papoular 1990; David \& Pégourié 1995), among which this ratio ranges from 0.28 to 0.50 (see Ossenkopf et al. 1992) for more detailed discussion of each data set). The dust emissivity in the circumstellar shell can be derived in a statistical way based on a large sample of stars, such as the IRAS LRS database, but either the inner dust shell temperature (Volk \& Kwok 1988), the $18 \mu \mathrm{m}$ to $10 \mu \mathrm{m}$ band ratio (Simpson 1991), or the inner shell radius (Marengo et al. 1997) still has to be assumed in the derivation.

The inner dust shell temperature has, in fact, been one of the important issues in the dust shell studies and should be a crucial parameter in the investigation of the dust formation process. Chemical equilibrium calculations indicate a condensation temperature around $1200 \mathrm{~K}$ for silicate grains (Salpeter 1977), while the classical nucleation theory suggests that the condensation occurs around 1000 K (Draine 1981; Kozasa et al. 1984). Condensation around oxygen-rich stars may involve complicated chemistry because no simple monomers of the silicate composition are expected to exist in the gas phase. A recent more sophisticated model of heterogeneous condensation around M-type stars suggests that most dust is condensed in the region of $T=800-1000 \mathrm{~K}$ for a large mass-loss case (Gail \& Sedlmayr 1999). Some of the observational studies of the dust shell have indicated much lower inner dust shell temperatures $(<700 \mathrm{~K})$ than these theoretical predictions, particularly for thin dust shells (Rowan-Robinson \& Harris 1982, 1983; Onaka et al. 1989a). However, it cannot be ruled out that the uncertainties in the adopted optical properties may result in the low temperatures. Recent interferometric observations have suggested that the inner dust shell is located at the region with temperatures around $1000 \mathrm{~K}$ for $o$ Cet and $850 \mathrm{~K}$ to $1170 \mathrm{~K}$ for R Aqr although the infrared spectrum from the fitted models did not show a satisfactory agreement with the observed spectra (Danchi et al. 1994; Tuthill et al. 2000). Episodic mass loss in AGB stars has been suggested 
by several observations (Izumiura et al. 1997; Hashimoto et al. 1998) and it could also lead to low inner dust shell temperatures.

It is difficult to fix the inner dust shell temperature either from observations or theories with accuracy at present stage and the determination of the dust properties has to be made in a different way. In this paper we propose a method to derive the optical properties that consistently explain the variabilities both in the dust temperature and the dust shell flux and apply it for the observations of $\mathrm{Z}$ Cyg. We investigate whether the observed variation can be interpreted in terms of the light variation of the central star and whether there is any evidence indicating the dust formation in the variability seen in the SWS spectrum. In Sect. 2, we present the observational results and describe the method of analysis in Sect. 3. Comparison with previous studies and implications on the dust formation are discussed in Sect. 4.

\section{Observations}

The target stars of the time variability program have been selected on the criteria of the infrared brightness and the visibility of ISO. Z Cyg is a Mira variable star of M5e-M9e with the variability period of 264 days (Kholopov et al. 1985-1988). The IRAS LRS spectrum of Z Cyg clearly shows the 10 and $18 \mu \mathrm{m}$ silicate features (Joint IRAS Science Working Group 1986). Previous studies of decomposition of the spectra into two components suggest that the mid-infrared spectrum of Z Cyg is located in the extreme end of the variety and it represents a spectrum composed of only the silicate component with no or a very small amount of the aluminum oxide component (Onaka et al. 1989b; Miyata et al. 2000). Z Cyg is also known to have a very large negative LSR velocity $\left(-147.7 \mathrm{~km} \mathrm{~s}^{-1}\right)$ and a small terminal velocity $\left(4.0 \mathrm{~km} \mathrm{~s}^{-1}\right)$ in the CO radio emission (Young 1995). The mass-loss rate estimated from the $\mathrm{CO}$ observation is $4 \times 10^{-8} M_{\odot} \mathrm{yr}^{-1}$ (Young 1995), while the optical depth at $10 \mu \mathrm{m}$ derived from the dust shell model is suggested to be about 0.01 (Onaka et al. 1989b), indicating that Z Cyg is surrounded by an optically thin circumstellar shell.

Z Cyg was located in a good visibility zone of ISO and was observed 7 times with an interval of the variability phase of approximately 0.2 except for the last observation, which had about 0.3 phase interval from the previous observation. The observations of Z Cyg started near a minimum and covered the two following maxima. The observation dates and variability phases $\phi$ are listed in Table 1 , where $\phi=0$ and 1 correspond to maximum and the phase is counted beyond unity to indicate the consecutive nature of the observations. The visual light curve of $\mathrm{Z}$ Cyg is slightly asymmetric and has a minimum around $\phi=0.6$. The observations were made in the SWS01 mode (full grating scan for $2.38-45.2 \mu \mathrm{m}$ ) with the speed of 2 except for the first observation, in which the speed was set as 1 . These observations provided a spectral resolution of approximately 300-500. The spectrum taken
Table 1. Observation date and phase of Z Cyg.

\begin{tabular}{lc}
\hline \hline Observation date & Variability phase $\phi^{1}$ \\
\hline 1996 August 5 & 0.55 \\
1996 October 8 & 0.79 \\
1996 November 24 & 0.97 \\
1997 January 24 & 1.20 \\
1997 March 21 & 1.42 \\
1997 May 15 & 1.63 \\
1997 August 8 & 1.93 \\
\hline
\end{tabular}

${ }^{1}$ The variability phases are estimated from the AAVSO data.

at the last observation had missing parts in the range 2.6-3.0 $\mu \mathrm{m}$ (band $1 \mathrm{~b}$ ) and $12.6-15.0 \mu \mathrm{m}$ (band $3 \mathrm{a}$ ) due to telemetry trouble. The missing parts are small and do not affect the present analysis. Together with the SWS observations broad-band photometry was carried out by PHT in the 3.6, 11.5, and $25 \mu \mathrm{m}$ bands (Lemke et al. 1996). At the first observation, far-infrared photometry with LWS (LWS02) was also attempted (Clegg et al. 1996), but the data did not have a sufficient signal-to-noise ratio. In the later observations the LWS photometry was not executed.

The Off-Line Processing (OLP) version 10.1 data were used for the SWS observations. The SWS spectra were reduced by the Observers SWS Interactive Analysis Package (OSIA) version 2.0. ${ }^{1}$ The data of the PHT observations were reduced with the PHT Interactive Analysis (PIA) software package version $9.1,{ }^{2}$ in which both the calibration based on the internal fine calibration sources (FCSs) and the default calibration (Laureijs et al. 2001) were applied to estimate the internal accuracy. The final PHT data of Z Cyg were obtained by subtracting the background flux taken at 5 arcmin away from Z Cyg in the concatenated observations. Some of the SWS spectra show apparent gaps between the band boundaries. The band $3 \mathrm{a}$ often appears faint compared to the contiguous bands. We corrected the gaps by scaling the band flux referring to the band 1 flux, which has the least uncertainty in the flux calibration (Leech et al. 2001). Comparison with the PHT data confirms that the corrections do not introduce spurious effects (see Appendix A).

Figure 1 shows the SWS spectra of Z Cyg taken at 7 different phases. Large variations in the infrared region are clearly seen with the visual variability. In this paper we concentrate on the dust emission for $\lambda>7 \mu \mathrm{m}$. The spectra of the range $2.4-7 \mu \mathrm{m}$ also show significant variations, which provide information on the physical properties of the photosphere as well as the outer atmosphere.

1 The OSIA is a joint development of the SWS consortium. Contributing institutes are SRON, MPE, KUL and the ESA Astrophysics Division.

2 The PIA is a joint development by the ESA Astrophysics Division and the ISOPHT Consortium with the collaboration of the Infrared and Analysis Center (IPAC). Contributing ISOPHT Consortium institutes are DIAS, RAL, AIP, MPIK, and MPIA. 


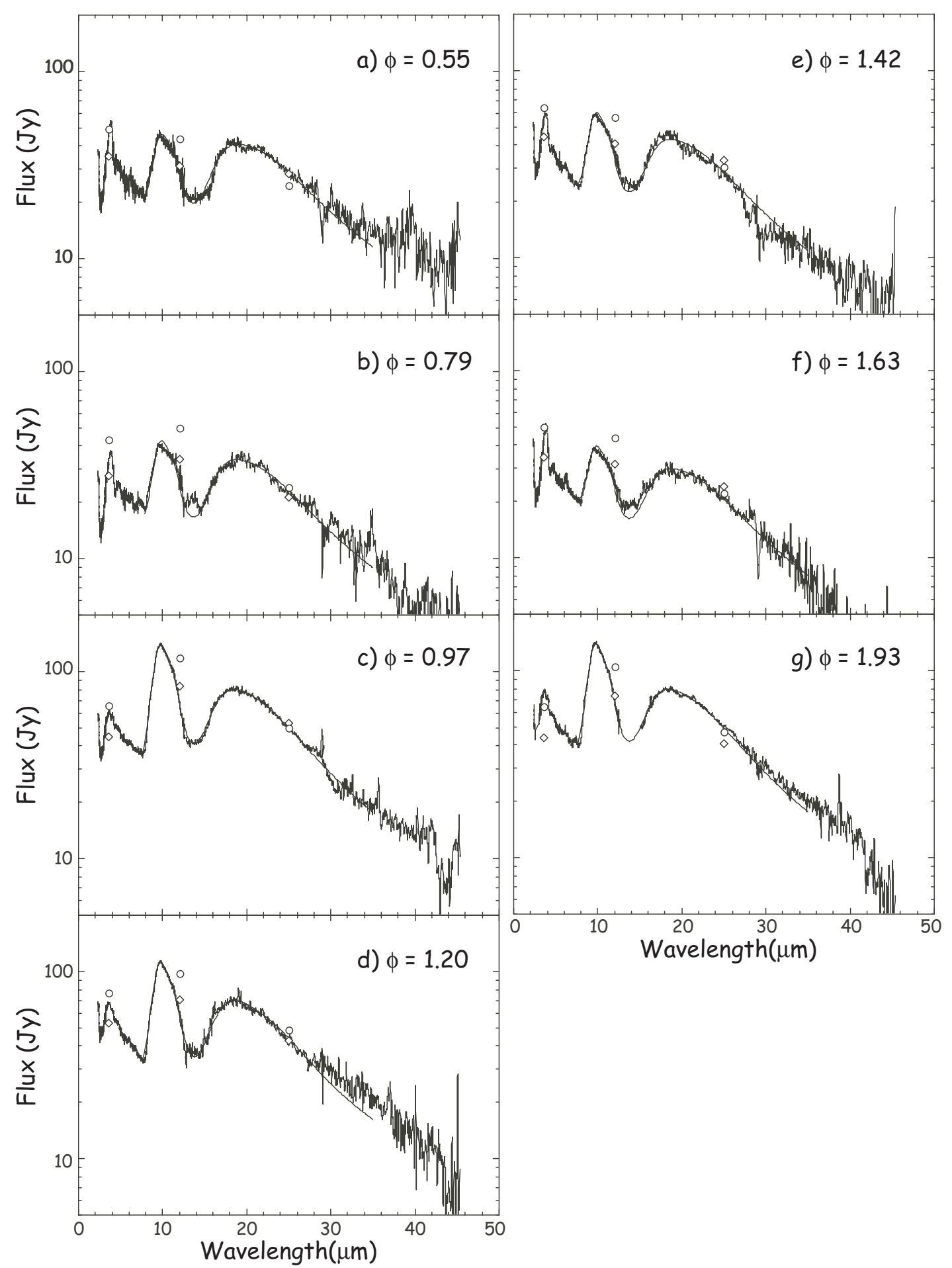

Fig. 1. Observed spectra of Z Cyg at 7 different epochs. The variability phase estimated from the visual light curve is indicated in each figure (Table 1). All the figures have the same scale. Open diamonds are the PHT data based on the default calibration, while open circles are those based on the FCSs. The thin smooth lines indicate the best fit model with $y_{0}=0.1$ and the dust emissivity $Q_{7}$ (see Sect. 3). The dust emissivity is derived from the spectrum at $\phi=0.97$ and thus the model fits perfectly with the observed spectrum.

Investigations on the short wavelength range are reported in Matsuura et al. (2002). The mid-infrared spectra of $\mathrm{Z}$ Cyg show a clear trend that the circumstellar emission in the 10-20 $\mu \mathrm{m}$ region increased relative to the photospheric emission of around $4 \mu \mathrm{m}$ from minimum to maximum. The ratio of the $10 \mu \mathrm{m}$ to $18 \mu \mathrm{m}$ bands also increased, indicating an increase in the dust temperature at maximum. The band ratio decreased as the star went to the second minimum (the variability phase $\phi=1.63$ ), where the spectrum became quite similar to the spectrum at the first minimum 
$(\phi=0.79)$. Then at the second maximum $(\phi=1.93)$ the star showed a spectrum nearly the same as that at the first maximum $(\phi=0.97)$. The variation in the infrared spectrum of Z Cyg appears to synchronize with the visual light variation.

The PHT data are plotted together in Fig. 1, taking account of the color corrections. We found that the SWS flux agrees with the PHT flux of the default calibration within $10 \%$ in most cases. The accuracy of the absolute flux calibration of SWS is estimated to be in a similar range $(12-15 \%$ for $\lambda>4 \mu \mathrm{m}$ at present, Leech et al. 2001). The general trends described above are also indicated by the PHT data, confirming the variations seen in the SWS spectra.

\section{Model analysis}

In the present study we attempt to derive the dust emissivity from the observed variation in the mid-infrared spectrum. If the dust emissivity $\kappa$ is proportional to $\lambda^{-\beta}$, where $\lambda$ is the wavelength and $\beta(>0)$ is a constant, the total flux from a dust grain $F_{\mathrm{gr}}$ is expected to vary as $F_{\text {gr }} \propto \int \kappa B_{\lambda}(T) \mathrm{d} \lambda \propto T^{4+\beta}$ with the temperature $T$, where $B_{\lambda}$ is the Planck function. Thus the variation in the shell flux is directly connected to the emissivity and to the variation in the dust temperature. In reality, the emissivity is a complicated function of the wavelength and the emergent dust shell flux is a summation of the emission from dust grains of different temperatures. To investigate the actual emissivity of the dust grains around Z Cyg we adopt a simple dust shell model. We assume that the dust shell is optically thin and spherically symmetric. The spectrum of the emergent dust shell flux $F(\lambda)$ is then given by

$F(\lambda)=4 \pi \int_{r_{\mathrm{i}}}^{r_{\mathrm{m}}} \sigma(\lambda) B_{\lambda}(T(r)) r^{2} n(r) \mathrm{d} r / D^{2}$,

where $\sigma(\lambda)$ is the emission cross-section of the dust grains, $T(r)$ is the temperature at the distance $r$ from the central star, $n(r)$ is the number density of the dust grains, $D$ is the distance from the earth to the star, and $r_{\mathrm{i}}$ and $r_{\mathrm{m}}$ are the inner and outer boundaries of the dust shell, respectively. We set $T\left(r_{\mathrm{m}}\right)=30 \mathrm{~K}$ and the inner dust shell temperature $T_{\mathrm{i}}=T\left(r_{\mathrm{i}}\right)$ as a free parameter in the model fitting. For spherical grains $\sigma(\lambda)$ can be written as $\pi a^{2} Q(\lambda)$, where $Q(\lambda)$ is the absorption efficiency factor or the dust emissivity, and $a$ is the grain radius. The efficiency factor $Q(\lambda)$ is proportional to $a$ for $a \ll \lambda$, which is a good approximation in the infrared range (van de Hulst 1957). As for the density distribution $n(r)$, we take into account the acceleration of the flow near the bottom of the envelope and adopt the "third simple model" of Habing et al. (1994):

$n(x)=n_{\mathrm{i}} / \sqrt{\left(x-y_{0}\right) x^{3}}$

with

$x=r / r_{\mathrm{i}} \quad$ and $\quad y_{0}=1-\left(v_{\mathrm{s}} / v_{\text {out }}\right)^{2}$, where $v_{\mathrm{s}}$ is the local sound velocity at $r_{\mathrm{i}}$ and $v_{\text {out }}$ is the terminal gas flow velocity. In this model, the dust velocity is assumed to be proportional to the gas velocity. This model provides a reasonable approximation to more accurate models in most cases (Habing et al. 1994). The parameter $y_{0}$ depends on the efficiency of the momentum transfer (e.g., Ivezić \& Elitzur 1995) and thus is a function of the mass-loss rate. In this paper we simply take it as a parameter and make calculations for the cases with $y_{0}=0,0.1$, and 0.4 . The parameter $y_{0}=0.1$ represents the most likely case for low mass-loss rates (Habing et al. 1994). We find that the results do not depend sensitively on the value of $y_{0}$ and thus we present results for $y_{0}=0.1$ in the followings unless explicitly indicated otherwise. Note that the case $y_{0}=0$ corresponds to a constant flow velocity model $\left(\rho \propto r^{-2}\right)$. The case $y_{0}=0.4$ provides an extreme situation whose density distribution deviates strongly from the case of constant flow velocity near the bottom of the shell.

The temperature of the grains at $r$ is calculated from the radiative equilibrium condition:

$$
\int_{0}^{\infty} Q(\lambda) F^{*}(\lambda)\left(r_{*} / r\right)^{2} \mathrm{~d} \lambda=4 \pi \int_{0}^{\infty} Q(\lambda) B_{\lambda}(T(r)) \mathrm{d} \lambda
$$

where $r_{*}$ is the stellar radius and $F^{*}(\lambda)$ is the photospheric flux of the star at $r_{*}$. The continuum around $4 \mu \mathrm{m}$ is assumed to be due to photospheric emission in the present analysis. The underlying photosphere should include the contribution from the extended atmosphere or the MOLsphere, which has been detected in several late-type stars (e.g., Tsuji et al. 1997; Tsuji 2000a, 2000b; Yamamura et al. 1999a, 1999b) and theoretically investigated in pulsating stars (Fleischer et al. 1992; Bessel et al. 1996; Höfner et al. 1998; Höfner 1999). This component is expected to show a complicated dependence on the visual variability and it is difficult to predict detailed spectra of the outer atmosphere at present (Lobel et al. 2000). Investigations of the near-infrared spectra suggest that the depression of the continuum due to absorption of water vapor in the outer atmosphere may be significant (up to $30 \%$ ) even at $4 \mu \mathrm{m}$ (Matsuura et al. 2002). In this paper we simply assume that the photospheric emission has a functional form with a stellar effective temperature $T^{*}$ given by Engelke (1992), which is scaled to the observed flux around $4 \mu \mathrm{m}$, and that most of the emission at $\lambda>7 \mu \mathrm{m}$ comes from the dust shell. The contribution from the photosphere is estimated to be less than $20 \%$ in the $10 \mu \mathrm{m}$ region even at minimum and the uncertainties in the subtraction procedure do not affect the present results significantly. In Eq. (4) the attenuation due to absorption by grains is neglected since in the present case the optical depth of the grains is still small in the near-infrared, where most of the stellar radiation energy resides, and since the effect is small compared to the uncertainty in the spectrum of the photospheric emission. For $T^{*}$ we adopt values of $2300 \mathrm{~K}, 2200 \mathrm{~K}, 2700 \mathrm{~K}, 2500 \mathrm{~K}$, $2300 \mathrm{~K}, 2200 \mathrm{~K}$, and $2700 \mathrm{~K}$ at phases $\phi=0.55,0.79,0.97$, $1.20,1.42,1.63 .1 .93$, respectively, according to the results 
for $o$ Cet by Danchi et al. (1994). These temperatures may not directly correspond to the photospheric temperatures of the star. The effect of uncertainties in the stellar temperatures is found to be insignificant in deriving the dust emission and it does not affect the present results.

Since the photospheric contribution becomes dominant for wavelengths shorter than $7 \mu \mathrm{m}$, the dust emissivity cannot be estimated from the observations accurately. The optical properties of the dust grains shorter than $7 \mu \mathrm{m}$ were calculated for astronomical silicate (Draine \& Lee 1984), assuming spherical dust grains with a radius of $0.1 \mu \mathrm{m}$. The absorptivity in the near-infrared determines the physical size of the dust shell, but does not affect the emergent spectrum because of the assumption of an optically thin dust shell. For the optical properties of dust grains at wavelengths longward of $7 \mu \mathrm{m}$, we created a set of dust absorption efficiency factors from the spectrum observed at the first maximum $(\phi=0.97)$ by changing the assumed inner dust shell temperature. From Eq. (1) the relative spectral shape of the absorption efficiency factor $Q(\lambda)$ is derived straightforwardly from the observed spectrum owing to the nature of the optically thin dust shell. The derived absorption efficiency factor was scaled such that $Q / a=1.35 \mu \mathrm{m}^{-1}$ at the peak around $9.7 \mu \mathrm{m}$ to agree with that of the astronomical silicate. This scaling was needed to make the optical properties connect smoothly at $7 \mu \mathrm{m}$. The absolute values of the absorption coefficient cannot be determined by the present analysis.

By adopting inner dust shell temperature of $400 \mathrm{~K}$, $500 \mathrm{~K}, 600 \mathrm{~K}, 700 \mathrm{~K}, 800 \mathrm{~K}, 900 \mathrm{~K}$, and $1000 \mathrm{~K}$ at the first observation maximum $(\phi=0.97)$, we have finally obtained a set of 7 dust absorption efficiency factors. We denoted them as $Q_{i}(i=4, \ldots 10)$. To remove the noise originating from the observed spectrum, we fitted $Q_{i}$ to the spectrum by using an analytical function. For spherical grains of $a \ll \lambda$, the absorption efficiency factor is given by

$Q(\lambda)=\frac{8 \pi a}{\lambda} \operatorname{Im}\left\{\frac{\epsilon(\lambda)-1}{\epsilon(\lambda)+2}\right\}$,

where $\epsilon(\lambda)$ is the complex dielectric constant of the dust (van de Hulst 1957). It can be approximated by a summation of oscillators which satisfy the dispersion relation (e.g., Koike et al. 1989):

$\epsilon=\epsilon_{\infty}+\sum_{j} \frac{2 P_{j} \Gamma_{j} \lambda^{2}}{\lambda_{j}} \frac{1}{\left(\lambda^{2}-\lambda_{j}^{2}\right)-i \Gamma_{j} \lambda_{j}}$,

where $\epsilon_{\infty}$ is the dielectric constant in the spectral range much shorter than the wavelengths in question and $P_{j}$, $\Gamma_{j}$, and $\lambda_{j}$ are the strength, width and position of each oscillator. We find that the emissivity derived from the observed spectrum can be fitted quite well with a set of five oscillators. The "best fit" parameters of the five oscillators are determined by least square fitting method. The efficiency factors used in the model fitting for $y_{0}=0.1$ are shown in Fig. 2. For other values of $y_{0}$, we derived similar $Q_{i}$ 's. The ratio of the $18 \mu \mathrm{m}$ to $10 \mu \mathrm{m}$ bands increases from $Q_{4}$ to $Q_{10}$ as expected.

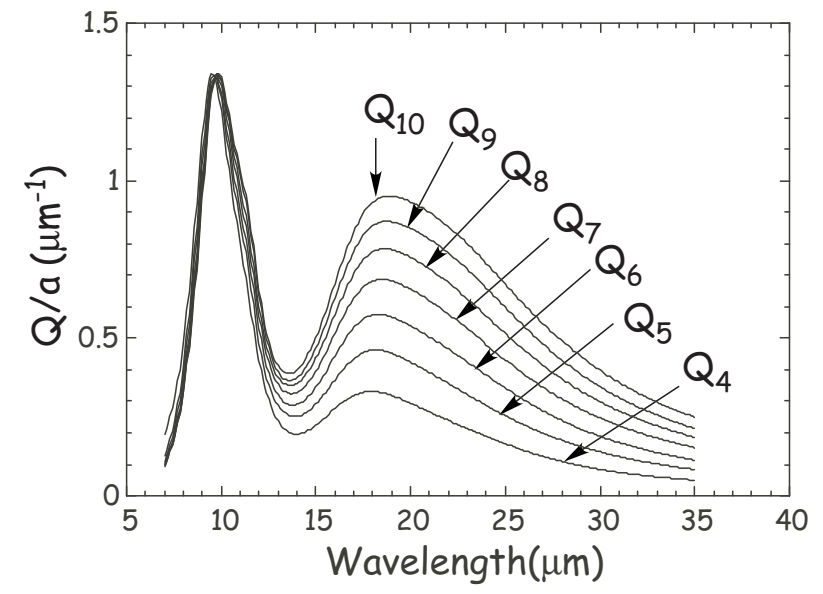

Fig. 2. Trial absorption efficiency factors for the models with $y_{0}=0.1$. Each curve labeled by $Q_{i}(i=4, \ldots 10)$ is derived with the assumed inner dust shell temperature at the first maximum $(\phi=0.97)$ of $400 \mathrm{~K}, 500 \mathrm{~K}, 600 \mathrm{~K}, 700 \mathrm{~K}, 800 \mathrm{~K}, 900 \mathrm{~K}$, and $1000 \mathrm{~K}$, respectively (see text).

With each $Q_{i}$ we fit all the observed spectra with the model, in which the inner dust shell temperatures $T_{\mathrm{i}}$ and the inner dust density $n_{\mathrm{i}}$ are the only free parameters. The best fit spectra with $y_{0}=0.1$ and $Q_{7}$ for each observed spectrum are superposed in Fig. 1. Figure 3 shows the parameters of the best fit models for $Q_{4}, Q_{7}$, and $Q_{10}$, where $n_{\mathrm{i}}$ is normalized to the maximum at $\phi=0.97$ for each $Q_{i}$ and $r_{\mathrm{i}}$ is assumed to not vary from phase to phase. The density $n_{\mathrm{i}}$ scales as $r_{\mathrm{i}}^{-3}$ if $r_{\mathrm{i}}$ changes (see Eq. (1)). Although we obtained different values of $T_{\mathrm{i}}$ and $n_{\mathrm{i}}$ for the best fit models with each $Q_{i}$, the best fit spectra appear to not differ from each other. All of them provide similarly good fits to the observations. Some examples are shown in Fig. 4 for $y_{0}=0.1$. The fitted spectra are almost identical to each other. Similar results are also obtained for the cases with $y_{0}=0$ and $y_{0}=0.4$. Therefore, we cannot distinguish between models with the dust emissivities and density distributions examined here solely from the quality of the fit.

Dust formation, if it occurs, adds new dust grains to the shell and could lead to a variation in the observed spectrum. However, dust formation is believed to occur mostly at minimum (e.g., Winters et al. 1994) and it is difficult to imagine that dust formation will explain the observed increase in the flux at maximum. The dust velocity near the bottom of the shell is several $\mathrm{km} \mathrm{s}^{-1}$ for optically thin shells (Habing et al. 1994) and during the interval of the observations the dust grains travel only several $10^{10} \mathrm{~m}$, which causes a decrease of only a few degrees in the dust temperature even at the bottom of the shell. This hardly affects the emergent spectrum. Furthermore, the motion of the dust is so slow that the decrease in the dust temperature from maximum to minimum cannot be due to removal of hot dust grains even if the observed change at maximum is attributed to the production of newly-formed hot dust grains near the bottom of the dust shell. Therefore we assume that the observed variations originate in the luminosity variation of the central star and that the density 


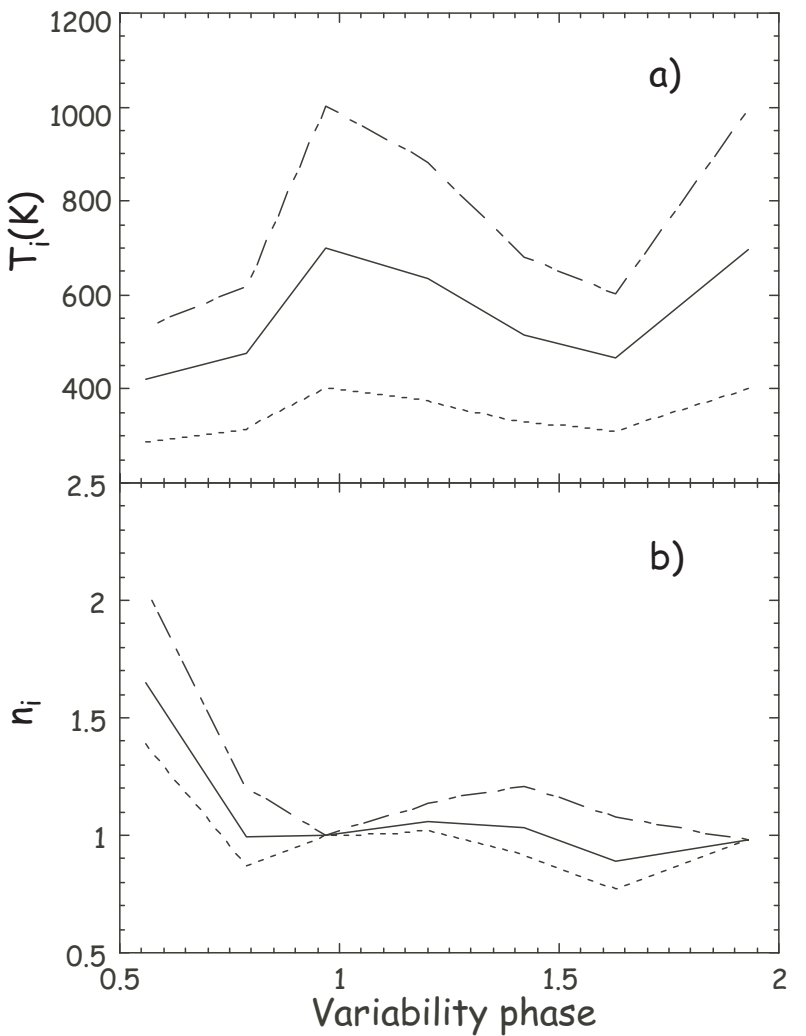

Fig. 3. Variations in the inner dust shell temperature $T_{\mathrm{i}}$ (a) and the normalized inner dust density $n_{\mathrm{i}}$ (b) for the "best fit" models with $y_{0}=0.1$. The solid lines represent the parameters for models with $Q_{7}$, the dotted lines those with $Q_{4}$, and the dot-dashed lines those with $Q_{10}$. The inner dust density is normalized to the visual maximum $(\phi=0.97)$ and is derived based on the assumption that the inner dust shell radius $r_{\mathrm{i}}$ is constant.

distribution does not change over the present observation period. Under these assumptions we investigate whether any of the seven dust emissivities can provide the flux variation compatible with the observations.

We directly use the observed dust shell flux to estimate the variation in the emergent flux. If the dust grains stay at the same position and the amount does not change, the variation in the dust shell flux at each variability phase can be predicted from the derived $T_{\mathrm{i}}$ and compared to the observations. We use the integrated flux of the observed dust shell from 5 to $45 \mu \mathrm{m}$ as the shell luminosity. There are some differences in the absolute flux levels between the SWS and PHT data (see Appendix A). In the present analysis we use the SWS flux to have consistency in the comparison of the model with the observations.

Figure 5 shows the comparison between the observations and the models with $Q_{4}, Q_{7}$, and $Q_{10}$ for $y_{0}=0.1$. In the models the amount of the dust grains in the shell is fixed for each $Q_{i}$ to provide the best match with all the observed spectra. We plot the uncertainties estimated from the quoted flux errors of the SWS spectra. The differences between the models are greatest at the maxima. If we take a high temperature dust model $\left(Q_{10}\right)$, the model

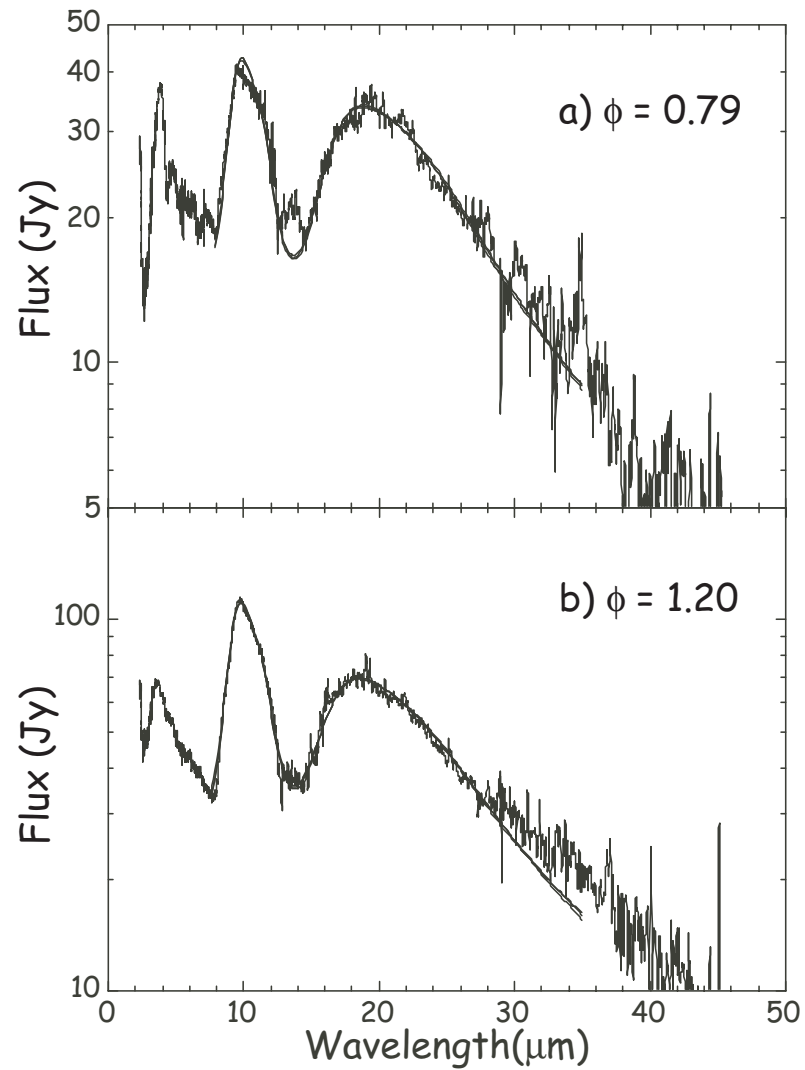

Fig. 4. Examples of the model fits to the spectra at $\phi=$ 0.79 (a) and 1.20 (b). The "best fit" models with $Q_{4}, Q_{7}$, and $Q_{10}$ are plotted by smooth thin lines together with the observed spectra, but they are hardly distinguishable from each other.

predicts too large a variation from minimum to maximum because it requires a large change in the dust temperature to explain the observed variation in the infrared spectral shape. In contrast the model of low dust temperature $\left(Q_{4}\right)$ shows too small a variation between minimum and maximum since a small change in $T_{\mathrm{i}}$ with $Q_{4}$ can explain the observed spectral variations.

To make a quantitative comparison we plot reduced $\chi^{2}$ values $\left(=\sum_{i=1}^{7}\left(f_{\text {model }, i}-f_{\text {obs }, i}\right)^{2} / \sigma_{i}^{2} / 6\right.$, where $f_{\text {model }, i}$ and $f_{\mathrm{obs}, i}$ are the model and observed fluxes, respectively, $\sigma_{i}$ is the uncertainty in the flux, and 6 is the number of degrees of freedom of the fit, the number of the observation epochs (7) minus the number of the dust amount scaling factor (1)) for each $Q_{i}$ model in Fig. 6. The emissivity $Q_{7}$ gives the smallest $\chi^{2}$ values for $y_{0}=0,0.1$, and 0.4 . The increase in $\chi^{2}$ in the high temperature models depends on the assumed dust optical properties in the $5-7 \mu \mathrm{m}$ region. We find that $\chi^{2}$ is minimum for the emissivity $Q_{7}$ unless the real dust properties differ by more than a factor of 3 from those of the assumed astronomical silicate. Even if we change the $5-7 \mu \mathrm{m}$ absorptivity by a factor of 10 , the minimum is shifted to either $Q_{6}$ or $Q_{8}$. Thus the present results are not sensitive to the absorptivity in $5-7 \mu \mathrm{m}$. The models with the emissivity $Q_{7}$ provide the most consistent results with the observed variations in the spectral shape 


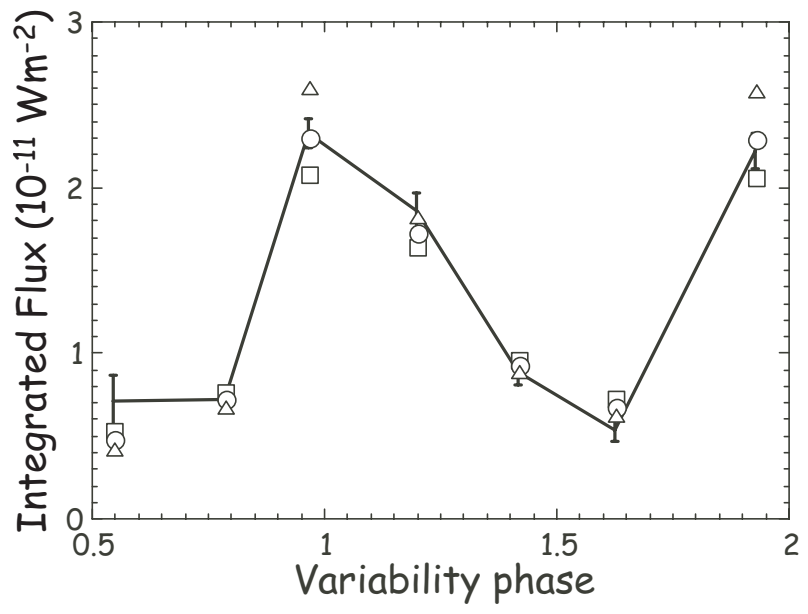

Fig. 5. The variation in the integrated dust shell flux in the range $5-45 \mu \mathrm{m}$. The solid line shows the observed variation. The open triangles are the results from the best fit models with $Q_{10}$, the open circles those with $Q_{7}$, and the open squares those with $Q_{4}$. The error bars indicate the uncertainties estimated from the SWS spectra and do not include the absolute calibration uncertainty.

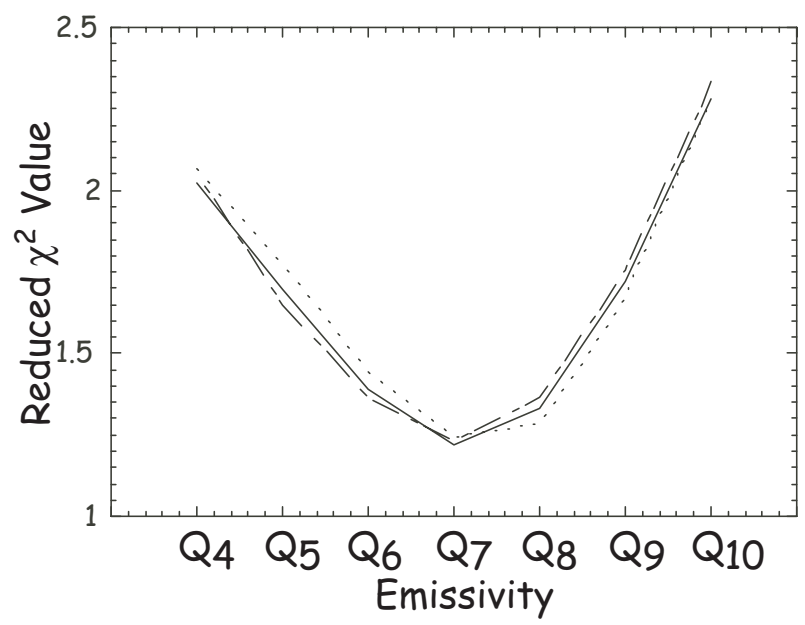

Fig. 6. Reduced $\chi^{2}$ values for the best fit models (see text for more details). The solid lines indicated the results for $y_{0}=0.1$, the dotted line for $y_{0}=0.4$, and the dot-dashed line for $y_{0}=0$.

$\left(T_{\mathrm{i}}\right)$ and the flux $\left(n_{\mathrm{i}}\right)$. This can also be inferred from Fig. 3, where $Q_{7}$ gives the least variation in $n_{\mathrm{i}}$ over the variability phase. The model with $Q_{7}$ predicts a low integrated flux at the first minimum $(\phi=0.55)$, which marginally agrees with the observed value, but it agrees with the observed integrated flux at the rest of the phases within the uncertainties. The discrepancy between $\phi=0.55$ and 0.79 will be discussed in terms of possible dust formation near minimum in the next section.

Models with $Q_{6}$ and $Q_{8}$ differ insignificantly from the $Q_{7}$ model in $\chi^{2}$ values. Comparison with the PHT data suggests that the SWS spectrum of the last observation $(\phi=1.93)$ may be systematically brighter by approximately $15-20 \%$ relative to the observations at the other phases (Appendix A). If we reduce the integrated flux of the last observation by $20 \%$, then we find that the $Q_{6}$ model gives a slightly smaller $\chi^{2}$ value in the integrated flux than the $Q_{7}$ model. In the spectrum fit we also find that a change in $T_{\mathrm{i}}$ by $10 \%$ increases $\chi^{2}$ by a factor of 2 , which corresponds to about $20 \%$ change in the predicted flux level. This changes the minimum in $\chi^{2}$ either to $Q_{6}$ or $Q_{8}$. Therefore, we estimate that the uncertainty in the determination of the most likely emissivity is about one division in the emissivity set $(100 \mathrm{~K})$ and conclude that the models with $T_{\mathrm{i}}=700 \pm 100 \mathrm{~K}$ at visual maximum provide the best fit to both the spectral shape and the flux level. The derived inner dust temperature at maximum is somewhat low compared to theoretical predictions, but is not significantly different from the range $800-1000 \mathrm{~K}$ suggested by the latest investigation (Gail \& Sedlmayr 1999).

The best fit for $Q_{7}$ implies a mass-loss rate $\dot{M}=$ $7 \times 10^{-8}\left(r_{*} / 3 \times 10^{13} \mathrm{~cm}\right)(D / 490 \mathrm{pc})^{2}\left(v_{\mathrm{i}} / 5 \mathrm{~km} \mathrm{~s}^{-1}\right)$ $(\delta / 200) M_{\odot}$, where $v_{\mathrm{i}}$ is the dust flow velocity at the inner shell boundary and $\delta$ is the gas to dust ratio. If we take $r_{*}=3 \times 10^{13} \mathrm{~cm}, \delta=200, v_{\mathrm{i}}=5 \mathrm{~km} \mathrm{~s}^{-1}$, and $D=490 \mathrm{pc}$ (Young 1995), the estimated mass-loss rate is in fairly good agreement with the results of the $\mathrm{CO}$ observations $\left(4 \times 10^{-8} M_{\odot}\right.$, Young 1995). The optical depth at $9.7 \mu \mathrm{m}$ is derived to be 0.08 . It is larger than the previous estimate (Onaka et al. 1989b) because the present study derives a higher $T\left(r_{\mathrm{i}}\right)$, but confirms that the optically thin approximation is still valid.

\section{Discussion}

The present observations of Z Cyg clearly show a large variation in the infrared spectrum: the circumstellar emission increases at visual maximum and the variation synchronizes fairly well with the visual light variation. Previous investigations of oxygen-rich Mira variables have suggested a similar enhancement in the $10 \mu \mathrm{m}$ silicate band around visual maximum in several stars (Hron \& Aringer 1994; Little-Marenin et al. 1996; Creech-Eakman et al. 1997; Miyata 1998; Monnier et al. 1998). CreechEakman et al. (1997) have suggested a change in the dust emissivity at maximum and attributed it to a change in the dust size distribution. Based on the ground-based observations, Monnier et al. (1998) have indicated that some M-type AGB stars show an enhancement in the $10 \mu \mathrm{m}$ band and suggested an associated sharpening of the band at maximum. The present observations were made over one variability period more frequently than previous studies owing to the good visibility of the ISO mission. The general trend seen for Z Cyg is in agreement with previous work, indicating that the present results may be representative for the variation in the infrared spectrum of this class of variables. In contrast to Creech-Eakman et al. (1997) and Monnier et al. (1998), however, the present analysis suggests that the silicate band profile does not change appreciably with variability phase and that the same dust emissivity can reproduce the observed spectra taken at different phases reasonably well. Based on detailed radiative-transfer model calculations, Lobel et al. (2000) have suggested that the observations of $o$ Cet by 


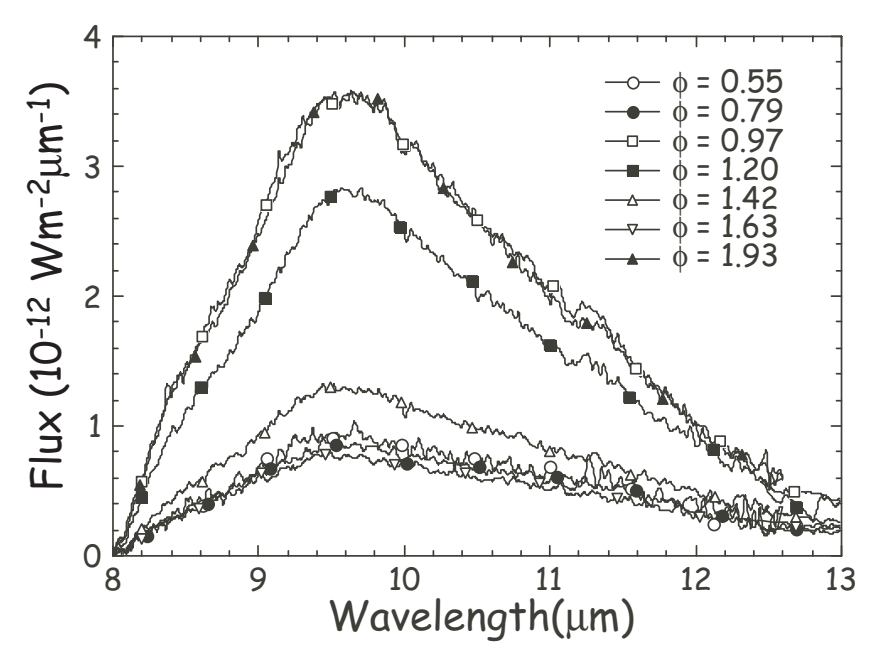

Fig. 7. The dust feature spectra of Z Cyg, in which the stellar continuum has been subtraced in the same way as in Monnier et al. (1998). The denotations of each spectrum are shown in the figure.

Monnier et al. (1998) can be interpreted in terms of the luminosity variation of the central star without any variation in the dust properties, in agreement with the present results.

Figure 7 shows a plot similar to that by Monnier et al. (1998). The stellar continuum has been subtracted in the same manner: the stellar continuum is assumed to be a $2500 \mathrm{~K}$ blackbody and the intensity is fitted at $8 \mu \mathrm{m}$. The intensity at the band peak is enhanced more at maximum than that at $12 \mu \mathrm{m}$, a result similar to Monnier et al. (1998). Figure 7 also confirms that the spectral profile varies systematically with phase. The apparent sharpening of the feature in Fig. 7 is interpreted in terms of the change in the dust temperature in the present analysis. Lobel et al. (2000) suggested that the observed sharpening results from the variation in the dust shell opacity due to the modulation in the mass-loss rate. Based on the silicate and aluminum oxide two-component model, Miyata (1998) has suggested that only the silicate component varies with the visual variation, but the aluminum oxide component does not. The variations seen in the $10 \mu \mathrm{m}$ band profile in the sample of Monnier et al. (1998) may result from the variation in the silicate component if the dust consists of more than one components. Then the present results suggest that the silicate component does not change its profile appreciably with the visual variation.

Although the general shape of the spectra is reproduced by the same dust emissivity quite well, there are some deviations in individual spectra (Fig. 1). There is an excess feature around $13 \mu \mathrm{m}$ at $\phi=0.79$. A similar excess is also seen at $\phi=1.63$ and thus this seems to be a real feature around minimum. This feature may be related to the $13 \mu \mathrm{m}$ feature seen in other oxygen-rich Mira variables (Sloan et al. 1996). The feature in Z Cyg seems to be broader than the $13 \mu \mathrm{m}$ feature but the low signalto-noise ratio together with the strong underlying continuum makes a precise comparison difficult. The $13 \mu \mathrm{m}$ feature has been proposed to originate from a high temperature condensate of aluminum oxide (Kozasa \& Sogawa 1997; Begemann et al. 1997), titanium oxide, spinel grains (Posch et al. 1999; Fabian et al. 2001), silicon dioxide or highly polymerized silicates (Speck et al. 2000). All of these materials should have additional weak features at $\lambda>15 \mu \mathrm{m}$. Those are not visible in the present spectra, but may be masked by the strong $18 \mu \mathrm{m}$ silicate band. If the presence of the $13 \mu \mathrm{m}$ feature in $\mathrm{Z}$ Cyg at minimum is confirmed, the present results suggest that the grains responsible for the $13 \mu \mathrm{m}$ feature behave differently with variability and therefore must be a separate component.

Some deviations can also be seen around the peak of the $10 \mu \mathrm{m}$ band at $\phi=0.79$ and 1.63 . The peak in the model spectra is somewhat sharper than in the observed spectra. Hron et al. (1997) have suggested that there should be variations in the $10 \mu \mathrm{m}$ region due to the molecular absorption in the outer atmosphere of Mira variables. Part of the observed variations in Z Cyg could be attributed to variations in the underlying continuum of the photosphere or of the outer atmosphere of the star. The spectrum at $\phi=0.55$ indicates a depression in the range $16-18 \mu \mathrm{m}$ relative to the model spectrum. This may also be attributable to variations in the underlying outer atmosphere emission. A similar depression is not clearly seen at other phases. There are some deviations seen in the $20 \mu \mathrm{m}$ band ( $\phi=1.42$ and 1.63$)$ and also in the region longward of $30 \mu \mathrm{m}$, but the low signal-to-noise ratio prevents further analysis.

Near-infrared observations indicate that a typical variation amplitude in the bolometric luminosity of Mira variables is in the range of a factor of 3-5 (Catchpole et al. 1979). The observed variation in the integrated infrared flux of Z Cyg has an amplitude of a factor of 3.3 (Fig. 5), suggesting that the luminosity variation of the central star can account for the variation in the infrared flux. The variation in the dust temperature results from the luminosity variation. However, there exists an apparent discrepancy between the first two observations. The observed spectra indicate an increase in the temperature from $\phi=0.55$ to 0.79 , but the flux level does not change accordingly. The discrepancy may still be within the range of the flux uncertainties because a comparison with the PHT data suggests that the SWS flux at $\phi=0.55$ may be overestimated by approximately $10 \%$ relative to the other phases (see Appendix A). On the other hand, this could also be the result of dust formation. If new dust grains are formed at $\phi=0.79$ we can expect more grains of high temperature near the inner shell radius at this epoch and the dust emission could become hotter.

To investigate the effects of possible dust formation on the emergent spectrum, we make a simple calculation. We assume that the dust grains are formed only near minimum and their amount is equal to that integrated over one variability cycle. Then the emission from the newlyformed dust grains $F_{\text {new }}(\lambda)$ can be given by

$F_{\text {new }}(\lambda)=4 \pi n_{\mathrm{i}} v_{\mathrm{i}} r_{\mathrm{i}}^{2} P \sigma(\lambda) B_{\lambda}\left(T_{\mathrm{f}}\right) / D^{2}$, 


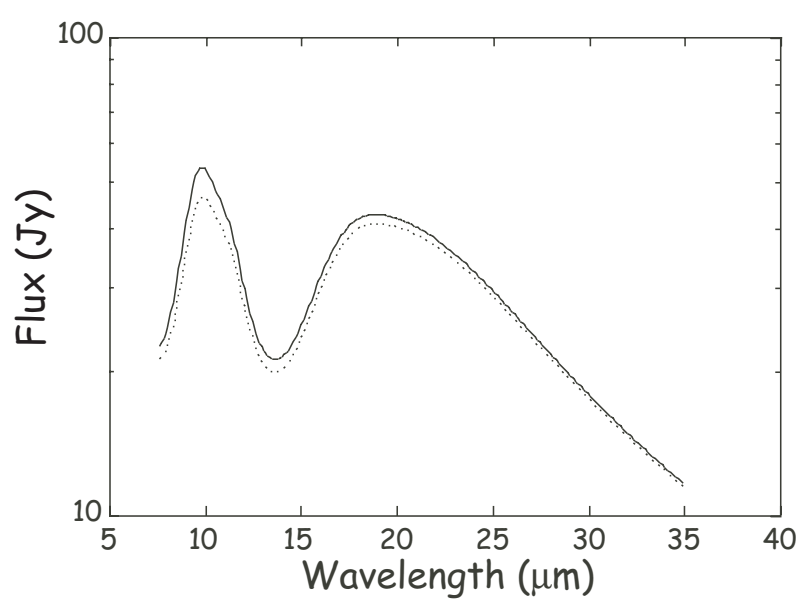

Fig. 8. Effects of possible dust formation. The dotted line indicates the "best fit" spectrum at $\phi=0.55$ with $Q_{7}$ and the solid line represents the spectrum in which the emission from newly-formed dust grains is added (see text).

where $P$ is the period of the variability (264 days), $v_{\mathrm{i}}$ is the dust flow velocity at the inner boundary of the dust shell, and $T_{\mathrm{f}}$ is the dust temperature at formation. We use the fit parameters at $\phi=0.97$ for $\left(n_{\mathrm{i}} / D^{2}\right)$ and $\left(r_{\mathrm{i}} / r_{*}\right)$, and assume $v_{\mathrm{i}}=5 \mathrm{~km} \mathrm{~s}^{-1}, r_{*}=3 \times 10^{13} \mathrm{~cm}$, and $T_{\mathrm{f}}=700 \mathrm{~K}$. Then $F_{\text {new }}(\lambda)$ is added to the model of $\phi=0.55$. The result is shown in Fig. 8. The effect is not large, though not negligible. The increase in the intergrated flux between $5-45 \mu \mathrm{m}$ is only $9 \times 10^{-13} \mathrm{~W} \mathrm{~m}^{-2}$ and will not affect the estimate of the dust shell luminosity. However, the increase in the $10 \mu \mathrm{m}$ flux relative to $20 \mu \mathrm{m}$ is obvious and the best fitted $T_{\mathrm{i}}$ changes from $421 \mathrm{~K}$ to $462 \mathrm{~K}$ for $Q_{7}$ and becomes close to $T_{\mathrm{i}}$ of $\phi=0.79(477 \mathrm{~K})$. Thus this may account for part of the increase in $T_{\mathrm{i}}$ at $\phi=0.79$. A similar deviation does not seem to be observed around the second minimum so that a repetition of this phenomenon could not be confirmed. The density in the inner part of the dust shell could also be affected by the shock propagation. While Fig. 8 suggests that the effects of local variations in the density profile on the dust shell emission are not very significant, further studies of detailed modeling taking account of the dynamical motion and of dust formation (cf., Winters et al. 2000; Simis et al. 2001) as well as observations around minimum would be quite interesting to investigate possible dust formation process and effects of the shock in detail. Interferometric observations will also be valuable to study dust formation in the circumstellar region (Onaka et al. 2001).

In the present analysis the absorption properties of dust grains in the near infrared are fixed. Also the photospheric emission is approximated by a simple function and the stellar temperature is assumed rather arbitrarily. However, since we use the infrared integrated flux rather than the stellar luminosity to estimate the incident radiation variation, these assumptions have little effect on the derived results. The integrated infrared flux simply represents the radiation absorbed by the dust grains and it is directly related to the dust temperature. The change

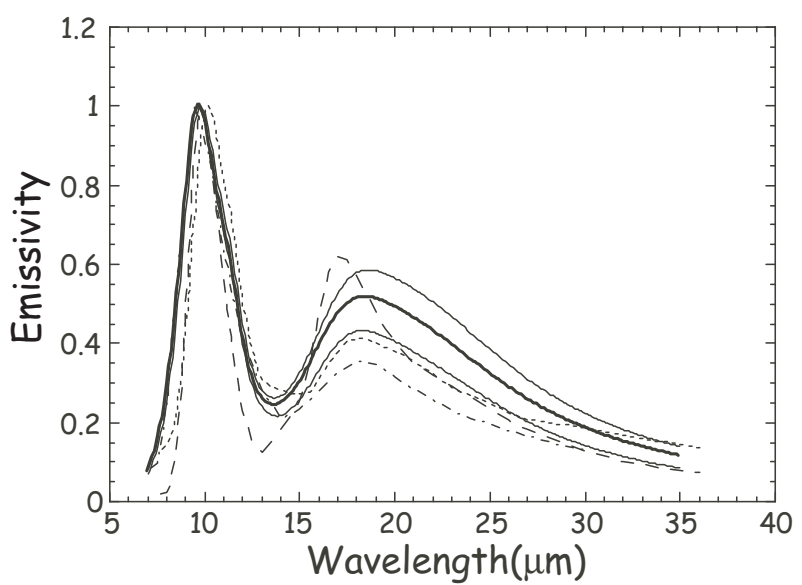

Fig. 9. Comparison of various dust emissivities with the present results. All the emissivities are normalized at their peak. The thick solid line indicates $Q_{7}$ and thin solid lines $Q_{6}$ and $Q_{8}$ derived for $y_{0}=0.1$ in the present analysis (see text). The dotted line indicates those of circumstellar dust (set 1) of Ossenkopf et al. (1992) and the dot-dashed line those of "astronomical silicate" by Draine \& Lee (1984). The dashed line show the emissivity of $\mathrm{MgFeSiO}_{4}$ grains (Dorschner et al. 1995).

in the absorptivity or the stellar emission will change the absolute value of the distance from the star for a given dust temperature, but the emergent spectrum will not be much affected because the dust shell is optically thin.

Laboratory experiments indicate that the silicate emissivity changes with temperature (Day 1976). Henning \& Mutschke (1997), however, suggest that the temperature effects are relatively weak for silicates (less than $10 \%$ from $300 \mathrm{~K}$ to $10 \mathrm{~K}$ ). While it is difficult to completely rule out the possibility, the variation of the emissivity with temperature might result in a different band profile. The fact that all observed spectra can be fitted by the same dust emissivity suggests that the temperature effect does not play a dominant role in the observed variations.

Figure 9 shows a comparison of the dust emissivity derived in the present study with previous work. All emissivities are normalized at their peak. The emissivities $Q_{7}$ (thick solid line) as well as $Q_{6}$ and $Q_{8}$ (thin solid lines) are plotted to indicate the range of the most likely dust emissivity around Z Cyg. The absorption efficiency factor $Q_{7}$ and the dielectric constants derived from Eqs. (5) and (6) are listed in Appendix B. In Fig. 9 the emissivity of astronomical silicate (Draine \& Lee 1984) is plotted by a dot-dashed line. The emissivity of circumstellar silicate grains (set 1) of Ossenkopf et al. (1992) is shown by a dotted line. The emissivity of $\mathrm{Z}$ Cyg dust has a peak at a slightly longer wavelength, but the profile is quite similar to "astronomical silicate" in the $10 \mu \mathrm{m}$ region. The optical properties of astronomical silicate have been derived from the interstellar dust emission while the peak wavelength of the interstellar dust is known to be slightly shorter than that of the circumstellar dust (Ossenkopf et al. 1992). The peak of the circumstellar dust emissivity in the $10 \mu \mathrm{m}$ 
region of Ossenkopf et al. (1992) seems to be shifted to longer wavelengths relative to the $\mathrm{Z} \mathrm{Cyg}$ dust. There is also a clear difference in the $20-30 \mu \mathrm{m}$ region. The $\mathrm{Z}$ Cyg dust has a broad hump around $20-25 \mu \mathrm{m}$ in addition to the peak around $18 \mu \mathrm{m}$. The presence of this feature seems to be secure since all the observed spectra require this component. A similar broad feature has been suggested in young stars and attributed to $\mathrm{FeO}$ (Molster et al. 1999; Demyk et al. 1999; van den Ancker et al. 2000). Arendt et al. (1999) have indicated the presence of a broad $22 \mu \mathrm{m}$ feature in the Cas A supernova remnant and attributed it to $\mathrm{Mg}$ protosilicate. Chan \& Onaka (2000) have suggested a similar feature around $22 \mu \mathrm{m}$ to be present in the dust in $\mathrm{H}$ II regions and external galaxies. Since this feature in the $\mathrm{Z}$ Cyg dust is merged to the strong silicate feature, it is difficult to make a precise comparison with the interstellar feature. Further investigations are definitely needed to understand the origin of the $22 \mu \mathrm{m}$ feature.

Taking account of the possible range of the derived emissivities $\left(Q_{6}-Q_{8}\right)$, we obtain the ratio $Q(18 \mu \mathrm{m}) /$ $Q(10 \mu \mathrm{m})$ to be $0.51 \pm 0.08$. This value is located in the higher end of the range of proposed emissivities (Ossenkopf et al. 1992). However, laboratory measurements suggest that the band ratio around 0.5 is not unusual for amorphous or glassy silicates (Koike \& Hasegawa 1987; Henning \& Mutschke 1997). Henning et al. (1995) also suggested that the mixture of silicates with metal oxides increases the ratio. Dorschner et al. (1995) have shown that olivine glass $\left(\mathrm{Mg}_{2 \mathrm{y}} \mathrm{Fe}_{2-2 \mathrm{y}} \mathrm{SiO}_{4}\right)$ has a ratio as large as 0.7. For comparison, the emissivity of $\mathrm{MgFeSiO}_{4}$ grains is also plotted in Fig. 9. It has a $20 \mu \mathrm{m}$ peak at shorter wavelengths than the Z Cyg dust. Greenberg \& Li (1996) have suggested that the polarization spectrum of the Becklin-Neugebauer object in the mid-infrared region is best fitted by core-mantle grains whose silicate core has a large $20 \mu \mathrm{m}$ to $10 \mu \mathrm{m}$ band ratio, like $\mathrm{MgFeSiO}_{4}$ grains. The present results indicate that the silicate grains formed around Mira variables may provide a core for such grains.

Figure 10 shows the results of fitting models using the Z Cyg dust to two other AGB stars, o Ceti and RR Aql. Both spectra were taken from the ISO data archive and reduced in a similar way. The fitted model for the RR Aql spectrum clearly shows a discrepancy in the $12-16 \mu \mathrm{m}$ region, but reproduces the rest of the observed spectrum relatively well, particularly in the $20 \mu \mathrm{m}$ region. The emission around $12-16 \mu \mathrm{m}$ may come from other kinds of dust. Potential carriers of small and broad dust emission bands in the trough between the two silicate features are $\mathrm{Mg}$-Al-oxides, Ca-Al-oxides, or solid $\mathrm{TiO}_{2}$. The contribution from $\mathrm{CO}_{2}$ molecules may also be significant in this spectral range. The spectrum of $o$ Cet is obviously different from that calculated with the Z Cyg dust. It does not show the $22 \mu \mathrm{m}$ hump, and the $10 \mu \mathrm{m}$ peak seems to be shifted to longer wavelengths. There is excess emission for $\lambda>30 \mu \mathrm{m}$ in both stars, possibly due to a different dust component. It is clear that the $\mathrm{Z}$ Cyg dust cannot reproduce silicate features around all AGB stars with silicate emission. It is highly desirable to carry out similar

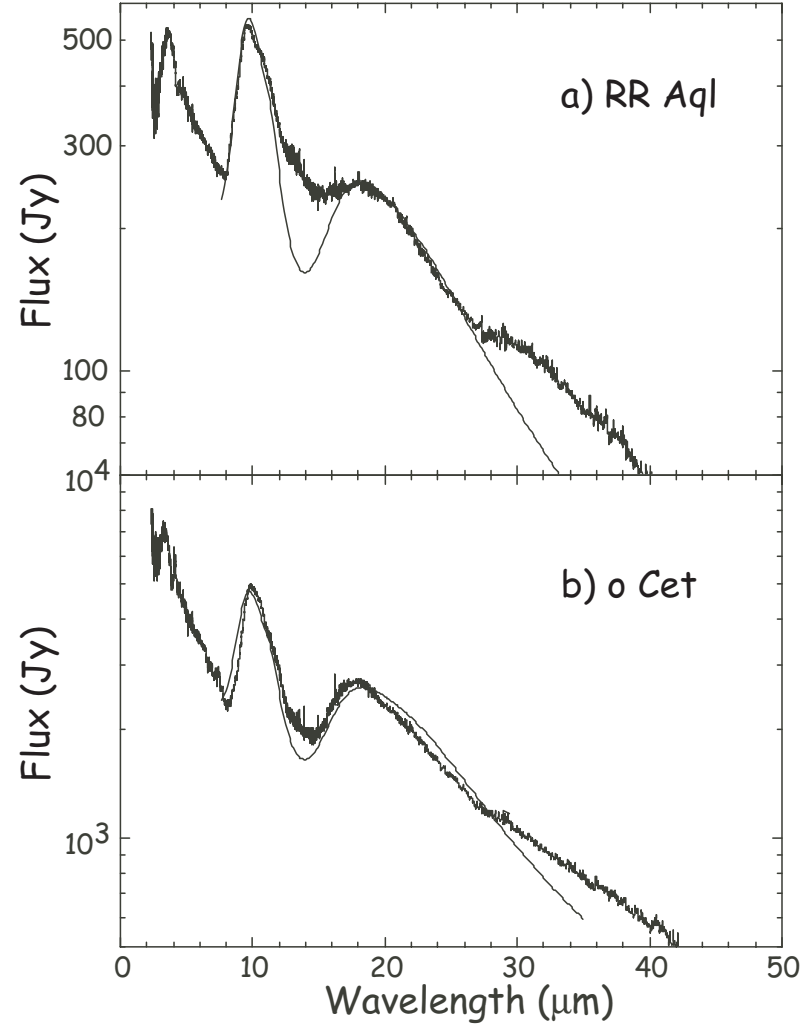

Fig. 10. Examples of the model fit with the Z Cyg dust emissivity for the SWS spectra of RR Aql (a) and o Cet (b). Thin solid lines indicate the model spectra

investigations on a larger sample and see whether the Z Cyg dust is able to reproduce the circumstellar silicate dust emission in the majority of Mira variables or not. The observations of the other target star of the present program, T Cep, show a quite different variation in its infrared spectrum (Onaka et al. 1999; Yamamura et al. 1999a), indicating that the variation of $Z$ Cyg indeed does not represent that of all Mira variables. Further observations of a larger sample of stars are definitely needed to better understand the general characteristics of the variation in the infrared spectrum of Mira variables.

\section{Conclusions}

The oxygen-rich Mira variable star, Z Cyg, has been observed with the SWS periodically over one and a half period. The SWS spectrum shows quite large variations. The circumstellar emission for $\lambda>7 \mu \mathrm{m}$ is enhanced and the ratio of the 10 to $18 \mu \mathrm{m}$ bands is increased around visual maxima. The variation can be interpreted in terms of the stellar luminosity variation if the proper dust emissivity is adopted. In other words, the dust emissivity can be derived by the spectroscopic variability observations. For optically thin dust shells, this analysis provides a fairly robust means to derive dust optical properties. Possible evidence for dust formation near minimum is discussed. The present analysis relies on the relation between $T_{\mathrm{i}}$ and the integrated flux, thus observationally on the spectral 
shape and the absolute flux level. Further improvement in the absolute calibration will enable a better determination of the dust optical properties. It will also facilitate detailed investigations of the dust formation process and the effects of dynamical motion of the dust shell. Studies of the photospheric emission of Mira variables taking account of the molecular opacities are also important to improve the estimation of the dust emissivity, particularly in the $10 \mu \mathrm{m}$ region. Application of this analysis to a large sample would be quite interesting to understand the general characteristics of the variability of oxygen-rich Mira variables in the infrared and the nature of the circumstellar dust grains. The optical properties derived for the Z Cyg dust shell indicate that the highest temperature of the dust shell is $700 \pm 100 \mathrm{~K}$ and the band ratio $Q(18 \mu \mathrm{m}) / Q(10 \mu \mathrm{m})$ is in the range $0.51 \pm 0.08$. The dust around $\mathrm{Z}$ Cyg is not representative for silicate dust around all oxygen-rich AGB stars, but it may be representative for a class of them. While dust emissivity variations in $10 \mu \mathrm{m}$ region have been indicated by previous studies, the present analysis suggests that such variations also exist in the $20 \mu \mathrm{m}$ region.

Acknowledgements. In this research, we have used, and acknowledge with thanks, data from the AAVSO International Database, based on observations submitted to the AAVSO by variable star observers worldwide. We would like to thank the SWS Instrument Dedicated Team (IDT) for their help in the data reduction, the ISO project members for their efforts in scheduling the time critical observations, and K. Kawara, Y. Satoh, T. Tanabé, H. Okuda, T. Tsuji, and other members of the Japanese ISO group for their continuous help and support. This work was supported in part by Grants-in-Aid for Scientific Research from the Japan Society of Promotion of Science (JSPS). I.Y. was supported by Grant-in-Aid for Encouragement of Young Scientists (No. 13740131) from the JSPS.

\section{Appendix A: Comparison between SWS and PHT data}

Figure A.1 show the plots of the PHT fluxes (3.6, 11.5, and $25 \mu \mathrm{m}$ ) at each variability phase together with the SWS fluxes (circles), which have been derived by convolving the SWS spectra with the PHT spectral sensitivity. Two PHT fluxes are plotted: one based on the default calibration (squares) and the other based on the internal fine calibration sources (FCSs; triangles). The quoted errors of the PHT data for each calibration are smaller than the size of the symbols in the plots. The SWS spectrum at the last observation had missing data in the $3.6 \mu \mathrm{m}$ and $11.5 \mu \mathrm{m}$ bands. For the $11.5 \mu \mathrm{m}$ band the model spectrum was used to complement the missing data. A simple interpolation was used to estimate the SWS flux for the missing part for the $3.6 \mu \mathrm{m}$ flux. Thus the uncertainties in these data may be larger than those at the other phases. The general trends seen in the SWS are confirmed by the PHT data. For the $3.6 \mu \mathrm{m}$ and $11.5 \mu \mathrm{m}$ bands, the PHT flux with the default calibration agrees fairly well with the SWS flux

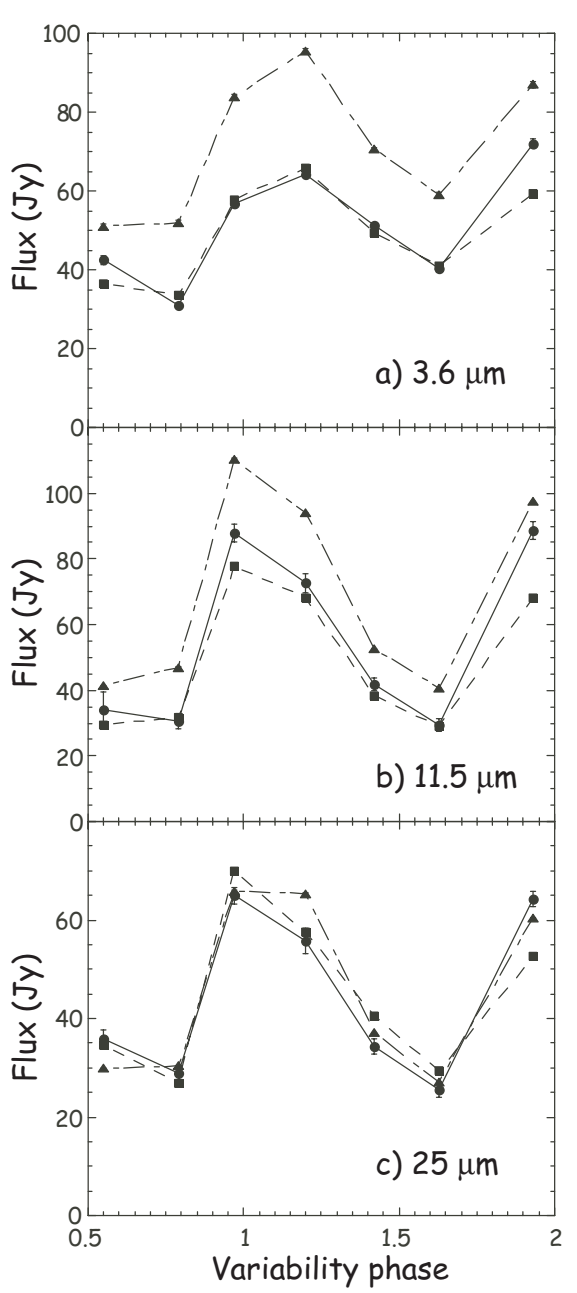

Fig. A.1. Comparison of the SWS flux with the PHT photometry of Z Cyg observations for $3.6 \mu \mathrm{m}$ (a), $11.5 \mu \mathrm{m}$ (b), and $25 \mu \mathrm{m}$. The SWS fluxes are derived by convolving the spectra with the PHT filter functions and indicated by filled circles connected by solid lines. The filled squares connected by dashed lines indicate the PHT data based on the default calibration, while the filled triangles with dot-dashed lines are derived based on the FCSs.

except for the last observation, while the PHT data with the FCSs always provide a larger flux than the SWS. The $25 \mu \mathrm{m}$ band flux of the SWS is also generally in better agreement with the PHT flux with the default calibration than that with the FCSs. For the last observation, all three band fluxes of the SWS are systematically larger by approximately $15-20 \%$ than the PHT fluxes relative to the observations of the other epochs. The SWS fluxes at the first phase $(\phi=0.55)$ also seem to be slightly large $(\sim 10 \%)$ relative to the other PHT fluxes, although that difference is still within the uncertainties.

\section{Appendix B: Table of the derived dielectric constants and emissivity}

In Table B.1 we list values of the complex refractive index $\epsilon=\epsilon_{1}+i \epsilon_{2}$ and of $Q / a$ for silicate dust derived from the "best fit" emissivity $Q_{7}$ for the case $y_{0}=0.1$. 
Table B.1. Dielectric constants and the emissivity $(Q / a)$ of the derived dust optical properties.

\begin{tabular}{|c|c|c|c|c|c|c|c|c|c|c|c|}
\hline $\begin{array}{l}\text { Wavelength } \\
(\mu \mathrm{m})\end{array}$ & $\overline{\epsilon_{1}}$ & $\overline{\epsilon_{2}}$ & $\begin{array}{c}Q / a \\
\left(\mu \mathrm{m}^{-1}\right)\end{array}$ & $\begin{array}{l}\text { Wavelength } \\
(\mu \mathrm{m})\end{array}$ & $\overline{\epsilon_{1}}$ & $\overline{\epsilon_{2}}$ & $\begin{array}{c}Q / a \\
\left(\mu \mathrm{m}^{-1}\right)\end{array}$ & $\begin{array}{l}\text { Wavelength } \\
(\mu \mathrm{m})\end{array}$ & $\overline{\bar{\epsilon}}$ & $\overline{\epsilon_{2}}$ & $\begin{array}{c}Q / a \\
\left(\mu \mathrm{m}^{-1}\right)\end{array}$ \\
\hline 7.6 & 0.434 & 0.130 & 0.218 & 16.8 & 0.512 & 0.986 & 0.608 & 26.0 & 1.573 & 2.584 & 0.385 \\
\hline 7.8 & 0.387 & 0.166 & 0.280 & 17.0 & 0.501 & 1.035 & 0.626 & 26.2 & 1.618 & 2.596 & 0.377 \\
\hline 8.0 & 0.338 & 0.208 & 0.356 & 17.2 & 0.493 & 1.083 & 0.643 & 26.4 & 1.663 & 2.607 & 0.368 \\
\hline 8.2 & 0.290 & 0.261 & 0.451 & 17.4 & 0.489 & 1.132 & 0.656 & 26.6 & 1.708 & 2.616 & 0.360 \\
\hline 8.4 & 0.242 & 0.323 & 0.566 & 17.6 & 0.488 & 1.181 & 0.667 & 26.8 & 1.753 & 2.624 & 0.352 \\
\hline 8.6 & 0.198 & 0.398 & 0.699 & 17.8 & 0.489 & 1.229 & 0.675 & 27.0 & 1.799 & 2.630 & 0.344 \\
\hline 8.8 & 0.161 & 0.486 & 0.848 & 18.0 & 0.494 & 1.276 & 0.681 & 27.2 & 1.843 & 2.634 & 0.336 \\
\hline 9.0 & 0.135 & 0.586 & 1.002 & 18.2 & 0.500 & 1.322 & 0.685 & 27.4 & 1.888 & 2.637 & 0.329 \\
\hline 9.2 & 0.125 & 0.698 & 1.144 & 18.4 & 0.509 & 1.367 & 0.686 & 27.6 & 1.932 & 2.638 & 0.321 \\
\hline 9.4 & 0.135 & 0.818 & 1.255 & 18.6 & 0.519 & 1.411 & 0.686 & 27.8 & 1.976 & 2.639 & 0.314 \\
\hline 9.6 & 0.170 & 0.940 & 1.320 & 18.8 & 0.530 & 1.453 & 0.685 & 28.0 & 2.019 & 2.637 & 0.307 \\
\hline 9.8 & 0.231 & 1.055 & 1.333 & 19.0 & 0.543 & 1.495 & 0.682 & 28.2 & 2.062 & 2.635 & 0.301 \\
\hline 10.0 & 0.315 & 1.154 & 1.301 & 19.2 & 0.557 & 1.535 & 0.678 & 28.4 & 2.104 & 2.631 & 0.294 \\
\hline 10.2 & 0.416 & 1.232 & 1.238 & 19.4 & 0.571 & 1.575 & 0.673 & 28.6 & 2.146 & 2.626 & 0.287 \\
\hline 10.4 & 0.523 & 1.283 & 1.161 & 19.6 & 0.587 & 1.613 & 0.668 & 28.8 & 2.187 & 2.620 & 0.281 \\
\hline 10.6 & 0.627 & 1.313 & 1.083 & 19.8 & 0.602 & 1.652 & 0.662 & 29.0 & 2.227 & 2.613 & 0.275 \\
\hline 10.8 & 0.721 & 1.328 & 1.011 & 20.0 & 0.619 & 1.689 & 0.656 & 29.2 & 2.266 & 2.605 & 0.269 \\
\hline 11.0 & 0.810 & 1.339 & 0.948 & 20.2 & 0.636 & 1.726 & 0.649 & 29.4 & 2.305 & 2.596 & 0.263 \\
\hline 11.2 & 0.902 & 1.351 & 0.888 & 20.4 & 0.654 & 1.763 & 0.642 & 29.6 & 2.343 & 2.586 & 0.258 \\
\hline 11.4 & 1.012 & 1.356 & 0.822 & 20.6 & 0.672 & 1.800 & 0.635 & 29.8 & 2.380 & 2.575 & 0.252 \\
\hline 11.6 & 1.140 & 1.329 & 0.743 & 20.8 & 0.691 & 1.837 & 0.627 & 30.0 & 2.416 & 2.563 & 0.247 \\
\hline 11.8 & 1.263 & 1.252 & 0.655 & 21.0 & 0.711 & 1.873 & 0.619 & 30.2 & 2.451 & 2.551 & 0.242 \\
\hline 12.0 & 1.346 & 1.137 & 0.572 & 21.2 & 0.732 & 1.910 & 0.611 & 30.4 & 2.486 & 2.538 & 0.237 \\
\hline 12.2 & 1.381 & 1.013 & 0.502 & 21.4 & 0.754 & 1.946 & 0.603 & 30.6 & 2.519 & 2.524 & 0.232 \\
\hline 12.4 & 1.378 & 0.900 & 0.448 & 21.6 & 0.777 & 1.982 & 0.594 & 30.8 & 2.552 & 2.510 & 0.227 \\
\hline 12.6 & 1.351 & 0.807 & 0.407 & 21.8 & 0.801 & 2.018 & 0.586 & 31.0 & 2.584 & 2.495 & 0.223 \\
\hline 12.8 & 1.310 & 0.733 & 0.376 & 22.0 & 0.826 & 2.054 & 0.577 & 31.2 & 2.615 & 2.479 & 0.218 \\
\hline 13.0 & 1.261 & 0.677 & 0.354 & 22.2 & 0.852 & 2.089 & 0.568 & 31.4 & 2.645 & 2.463 & 0.214 \\
\hline 13.2 & 1.209 & 0.634 & 0.339 & 22.4 & 0.880 & 2.124 & 0.558 & 31.6 & 2.674 & 2.447 & 0.210 \\
\hline 13.4 & 1.156 & 0.604 & 0.329 & 22.6 & 0.909 & 2.158 & 0.549 & 31.8 & 2.702 & 2.431 & 0.206 \\
\hline 13.6 & 1.102 & 0.583 & 0.325 & 22.8 & 0.939 & 2.192 & 0.539 & 32.0 & 2.730 & 2.414 & 0.202 \\
\hline 13.8 & 1.049 & 0.572 & 0.324 & 23.0 & 0.971 & 2.225 & 0.529 & 32.2 & 2.757 & 2.396 & 0.198 \\
\hline 14.0 & 0.997 & 0.567 & 0.328 & 23.2 & 1.004 & 2.258 & 0.520 & 32.4 & 2.783 & 2.379 & 0.194 \\
\hline 14.2 & 0.947 & 0.570 & 0.336 & 23.4 & 1.038 & 2.289 & 0.510 & 32.6 & 2.808 & 2.361 & 0.190 \\
\hline 14.4 & 0.898 & 0.578 & 0.346 & 23.6 & 1.074 & 2.320 & 0.500 & 32.8 & 2.832 & 2.343 & 0.187 \\
\hline 14.6 & 0.851 & 0.591 & 0.360 & 23.8 & 1.110 & 2.349 & 0.490 & 33.0 & 2.856 & 2.325 & 0.183 \\
\hline 14.8 & 0.806 & 0.610 & 0.377 & 24.0 & 1.148 & 2.378 & 0.480 & 33.2 & 2.878 & 2.307 & 0.180 \\
\hline 15.0 & 0.764 & 0.633 & 0.396 & 24.2 & 1.187 & 2.405 & 0.470 & 33.4 & 2.900 & 2.288 & 0.177 \\
\hline 15.2 & 0.724 & 0.660 & 0.417 & 24.4 & 1.227 & 2.431 & 0.460 & 33.6 & 2.922 & 2.270 & 0.173 \\
\hline 15.4 & 0.686 & 0.692 & 0.440 & 24.6 & 1.268 & 2.455 & 0.450 & 33.8 & 2.942 & 2.251 & 0.170 \\
\hline 15.6 & 0.652 & 0.726 & 0.464 & 24.8 & 1.310 & 2.478 & 0.441 & 34.0 & 2.962 & 2.233 & 0.167 \\
\hline 15.8 & 0.620 & 0.764 & 0.489 & 25.0 & 1.352 & 2.500 & 0.431 & 34.2 & 2.981 & 2.214 & 0.164 \\
\hline 16.0 & 0.592 & 0.804 & 0.515 & 25.2 & 1.395 & 2.520 & 0.422 & 34.4 & 3.000 & 2.196 & 0.161 \\
\hline 16.2 & 0.567 & 0.847 & 0.540 & 25.4 & 1.439 & 2.538 & 0.412 & 34.6 & 3.018 & 2.177 & 0.159 \\
\hline 16.4 & 0.545 & 0.892 & 0.564 & 25.6 & 1.483 & 2.555 & 0.403 & 34.8 & 3.035 & 2.159 & 0.156 \\
\hline 16.6 & 0.527 & 0.938 & 0.587 & 25.8 & 1.528 & 2.571 & 0.394 & 35.0 & 3.052 & 2.140 & 0.153 \\
\hline
\end{tabular}

\section{References}

Arendt, R. G., Dwek, E., \& Moseley, S. H. 1999, ApJ, 521, 234 Bedijn, P. J. 1987, A\&A, 186, 136

Begemann, B., Dorschner, J., Henning, Th., et al. 1997, ApJ, 476, 199

Bessell, M. S., Scholz, M., \& Wood, P. R. 1996, A\&A, 307, 481
Catchpole, R. M., Robertson, B. S. C., Lloyd Evans, T. H. H., et al. 1979, SSAO Circ., 1, 61

Chan, K.-W., \& Onaka, T. 2000, ApJ, 533, L33

Cheeseman, P., Stutz, J., Self, M., et al. 1989, Automatic Classification of Spectra from the Infrared Astronomical Satellite (IRAS) (NASA RP-1217) (Washington: GPO) 
Clegg, P., Ade, P. A. R., Armand, C., et al. 1996, A\&A, 315, L38

Creech-Eakman, M. J., Stencel, R. E., Williams, W. J., \& Klebe, D. I. 1997, ApJ, 477, 825

Danchi, W. C., Bester, M., Degiacomi, C. G., Greenhill, K. J., \& Townes, C. H. 1994, AJ, 107, 1469

David, P., \& Papoular, R. 1990, A\&A, 237, 425

David, P., \& Pégourié, B. 1995, A\&A, 293, 833

Day, K. L. 1976, ApJ, 203, L99

de Graauw, T., Haser, L. N., Beintema, D. A., et al. 1996, A\&A, 315, L49

Demyk, K., Jones, A. P., Dartois, E., Cox, P., \& d'Hendecourt, L. 1999, A\&A, 349, 267

Dorschner, J., Begemann, B., Henning, Th., Jäger, C., \& Mutschke, H. 1995, A\&A, 300, 503

Draine, B. T. 1981, in Physical Processes in Red Giants, ed. I. Iben Jr., \& A. Renzini (Reidel Publ. Co., Dordrecht), 317

Draine, B. T., \& Lee, H. M. 1984, ApJ, 285, 89

Engelke, C. W. 1992, AJ, 104, 1248

Fabian, D., Posch, Th., Mutschke, H., Kerschbaum, F., \& Dorschner, J. 2001, A\&A, 373, 1125

Ferrarotti, A. S., \& Gail, H.-P. 2001, A\&A, 371, 133

Fleischer, A. J., Gauger, A., \& Sedlmayr, E. 1992, A\&A, 266, 321

Gail, H.-P., \& Sedlmayr, E. 1999, A\&A, 347, 594

Greenberg, J. M., \& Li, A. 1996, A\&A, 309, 258

Habing, H. J., Tignon, J., \& Tielens, A. G. G. M. 1994, A\&A, 286,523

Hashimoto, O., Izumiura, H., Kester, D. J. M., \& Bontekoe, Tj. R. 1998, A\&A, 329, 213

Henning, Th., \& Mutschke, H. 1997, A\&A, 327, 743

Henning, Th., Begemann, B., Mutschke, H., \& Dorschner, J. 1995, A\&AS, 112, 143

Höfner, S. 1999, A\&A, 346, L9

Höfner, S., \& Dorfi, E. A. 1997, A\&A, 319, 648

Höfner, S., Jørgensen, U. G., Loidl, R., \& Aringer, B. 1998, A\&A, 340, 497

Hron, J., \& Aringer, B. 1994, A\&A, 281, 139

Hron, J., Loidl, R., \& Kerschbaum, F. 1997, Ap\&SS, 251, 211

Ivezić, Ž., \& Elitzur, M. 1995, ApJ, 445, 415

Izumiura, H., Waters, L. B. F. M., de Jong, T., et al. 1997, A\&A, 323, 449

Joint IRAS Science Working Group 1986, A\&AS, 65, 607

Kessler, M. F., Steinz, J. A., Anderegg, M. E., et al. 1996, A\&A, 315, L27

Kholopov, P. N., Samus', N. N., Frolov, M. S., et al. 1985-1988, General Catalogue of Variable Stars, 4th edition (Nauka Publishing House, Moscow)

Koike, C., \& Hasegawa, H. 1987, Ap\&SS, 134, 361

Koike, C., Hasegawa, H., Asada, N., \& Komatuzaki, T. 1989, MNRAS, 239, 127

Kozasa, T., \& Sogawa, H. 1997, Ap\&SS, 255, 437

Kozasa, T., Hasegawa, H., \& Seki, J. 1984, Ap\&SS, 98, 61

Laureijs, R. J., Klaas, U., Richards, P. J., Schulz, B., \& Ăbrahăm, P. 2001, The ISO Handbook V: PHT - The Imaging Photo-Polarimeter ver.1.2, ESA

Le Betre, T. 1993, A\&AS, 97, 729

Leech, K., de Graauw, T., van den Ancker, M., et al. 2001, The ISO Handbook Vol VI: SWS - The short wavelength spectrometer, ESA

Lemke, D., Klaas, U., Abolins, J., et al. 1996, A\&A, 315, L64

Le Sidaner, P., \& Le Bertre, T. 1993, A\&A, 278, 167
Little-Marenin, I. R., \& Little, S. J. 1988, ApJ, 333, 305

Little-Marenin, I. R., \& Little, S. J. 1990, AJ, 99, 1173

Little-Marenin, I. R., Stencel, R. E., \& Staley, S. B. 1996, ApJ, 467,806

Lobel, A., Bagnulo, S., Doyle, J. G., \& Power, C. 2000 , MNRAS, 317, 391

Marengo, M., Busso, M., Silvestro, G., Persi, P., \& Lagage, P. O. 1997, A\&A, 322, 924

Matsuura, M., Yamamura, I., Cami, J., Onaka, T., \& Murakami, H. 2002, A\&A, 383, 972

Miyata, T. 1998, Ph.D. Thesis, University of Tokyo

Miyata, T., Kataza, H., Okamoto, Y., Onaka, T., \& Yamashita, T. 2000, ApJ, 531, 917

Molster, F. J., Waters, L. B. F. M., Trams, N. R., et al. 1999, A\&A, 350, 163

Monnier, J. D., Geballe, T. R., \& Danchi, W. C. 1998, ApJ, 502,833

Monnier, J. D., Geballe, T. R., \& Danchi, W. C. 1999, ApJ, 521,261

Ohnaka, K., Balega, Y., Blöcker, et al. 2001, A\&A, 380, 212

Onaka, T., de Jong, T., \& Willems, F. J. 1989a, A\&A, 218, 169

Onaka, T., de Jong, T., \& Willems, F. J. 1989b, A\&AS, 142, 481

Onaka, T., de Jong, T., Yamamura, I., Cami, J., \& Tanabé, T. 1999, Proc. of The Universe as seen by ISO, ed. P. Cox, \& M. F. Kessler, ESA SP-427, 381

Ossenkopf, V., Henning, Th., \& Mathis, J. S. 1992, A\&A, 261, 567

Posch, T., Kerschbaum, F., Mutschke, H., et al. 1999, A\&A, 352,609

Rowan-Robinson, M., \& Harris, S. 1982, MNRAS, 200, 197

Rowan-Robinson, M., \& Harris, S. 1983, MNRAS, 202, 767

Salpeter, E. E. 1977, ARA\&A, 15, 267

Simis, Y. J. W., Icke, V., \& Dominik, C. 2001, A\&A, 371, 205

Simpson, J. P. 1991, ApJ, 368, 570

Sloan, G. C., \& Price, S. D. 1995, ApJ, 451, 758

Sloan, G. C., \& Price, S. D. 1998, ApJS, 119, 141

Sloan, G. C., LeVan, P. D., \& Little-Marenin, I. R. 1996, ApJ, 463,310

Speck, A. K., Barlow, M. J., Sylvester, R. J., \& Hofmeister, A. M. 2000, A\&AS, 146, 437

Stencel, R. E., Nuth, J. A. I., Little-Marenin, I. R., \& Little, S. J. 1990, ApJ, 350, L45

Tsuji, T. 2000a, ApJ, 538, 801

Tsuji, T. 2000b, ApJ, 540, L99

Tsuji, T., Ohnaka, K., Aoki, W., \& Yamamura, I. 1997, A\&A, 320, L1

Tuthill, P. G., Danchi, W. C., Hale, D. S., Monnier, J. D., \& Townes, C. H. 2000, ApJ, 534, 907

Volk, K., \& Kwok, S. 1988, ApJ, 331, 435

Vardya, M. S., de Jong, T., \& Willems, F. J. 1986, ApJ, 304, L29

van de Hulst, H. C. 1957, Light scattering by small particles (Dover Publications)

van den Ancker, M. E., Bouwman, J., Wesselius, P. R., et al. 2000, A\&A, 357, 325

Winters, J. M., Fleischer, A. J., Gauger, A., \& Sedlmayr, E. 1994, A\&A, 290, 623

Winters, J. M., Le Bertre, T., Jeong, K. S., Helling, Ch., \& Sedlmayr, E. 2000, A\&A, 361, 641

Yamamura, I., de Jong, T., Onaka, T., Cami, J., \& Waters, L. B. F. M. 1999a, A\&A, 341, L9

Yamamura, I., de Jong, T., \& Cami, J. 1999b, A\&A, 348, L55

Young, K., ApJ, 445, 872 